\title{
Ultrasound Detection of Explosives Using Wavelets for Synthesis of Features
}

\author{
Raycho Ilarionov*, Nikolay Shopov ${ }^{1}$, Ivan Simeonov and Hristo Kilifarev \\ Faculty of Electrical Engineering, Technical University of Gabrovo, \\ 4 H. Dimitar Str., 5300, Gabrovo, Bulgaria \\ 'University of Food Technologies, Plovdiv, 26 Maritsa Str., 4002, Plovdiv, Bulgaria
}

(Received July 27, 2009; accepted January 22, 2010)

Key words: explosive detection, wavelets, synthesis of features, ultrasound, ultrasonic sensors, noncontact

In the present paper, we propose a method of classifying Ammonite_ZH-B-E, Ammonite_E, and Trotyl by noncontact ultrasound acquisition of information. The received signals are processed using orthogonal wavelet basis functions of Haar (Daubechies 1), Daubechies, Coiflets, and Symlet. The application of this method in automatic classification systems is studied, focusing on the part concerned with the formation of feature complexes for assigning an explosive to a predefined class. By using discrete wavelet transforms (DWTs) with the above-mentioned orthogonal wavelets, the feature spaces of classifiers have been formed, which operate with a decision rule following the k-nearest neighbor (KNN) method. By using the classifiers thus synthesized, a test sample has been classified, and a very good result (overall precision, 98\%) was obtained when applying the Haar wavelet (Daubechies 1).

\section{Introduction}

The detection of explosive materials is a complex task that demands responsibility. Explosives are widely used in military science and practice, and also for civilian purposes, such as ore mining, road construction, and pyrotechnics. This requires the development and implementation of sensors and devices that can quickly generate precise and objective information on the type of explosive material. The development of such devices is difficult in view of the nature of the objects being classified - substances with high fire and explosion hazards. Moreover, the classification has to be conducted with sufficient speed and precision.

The requirements listed above lead to the development of

- noncontact sensors providing precise and objective information, and

- fast methods and algorithms for processing this information, and technical equipment for implementing them.

The synthesis of a system for the automatic classification of explosives is a

${ }^{*}$ Corresponding author: e-mail: hri-100@abv.bg 
multiaspect task. It involves the planning and conduct of experiments for collecting information, preparation of training and test samples, choosing a classifier, and reduction of recognition features, among others.

Special stationary and portable detectors have been developed for explosive detection. They usually analyze air samples or particles of the test material using different methods. ${ }^{(6,9)}$

Investigations have been conducted for three types of explosives - Ammonite_ZHB-E, Ammonite_E, and Trotyl — using ultrasound signals. A noncontact method for receiving primary information has been applied to the detection of these three explosives. Extraction and analysis of valuable information are the main tasks in the identification process. The analysis here is made even more difficult by the fact that the data sets are of high dimension (2500 discretes). One discrete consists of two values, namely, the time $(X$-axis) and voltage amplitude ( $Y$-axis) of the measured signal and represents the coordinates of one point from the oscillogram. These values also depend on the chosen settings for the horizontal and vertical scales and on the offset of the oscilloscope. The approach chosen during the tests is a strict mathematical formulation of the synthesis procedure of a feature complex for the recognition, and elimination of the effect of heuristic approaches using the general spectral theory of signals. The formation of a feature space is objectified, whose features are coefficients from the decomposition of the obtained samples in a predefined system of basis wavelet functions.

\section{Description}

Generally speaking, the chosen approach is based on a sound echolocation principle, whereby various materials can be recognized by the analysis of the ultrasound echo signal returned after reflection. The signal carries information on the characteristics of the material. ${ }^{(11)}$ The further processing of this signal is performed using algorithms for discrete wavelet transformation, reduction of classification attributes, and classification. According to the chosen classifier, the different explosives that are studied are classified as belonging to a certain class. The classes of objects have to be determined in advance on the basis of the available data.

The experiments for information acquisition have been conducted in an off-line mode using ultrasound sensors of the type UST40T/UST40R manufactured by Nippon Ceramic Company $^{(3)}$ (Fig. 1). A short sequence of ultrasound waves generated by a piezoelectric transmitter passes through the working medium (air, in this case). Upon reaching the material to be recognized, they are reflected by it. The returned signal is detected by the receiver. A typical oscillogram is presented in Fig. 2.

The discretization of the reflected signal is performed using the analogue-to-digital converter of the storage oscilloscope Tektronix TDS1002. The discretization frequency $\left(F_{S}\right)$ is $500 \mathrm{kHz}$. Each measurement yields 2500 samples. The data are recorded and transferred using the TDS2MEM Storage Memory and Communication Module (Fig. 1). Each measurement is stored in a separate file in CSV format.

The retrieval of useful information on the classification of an explosive as belonging to a certain class has been realized using spectral methods for image recognition,,$^{(1,4)}$ 


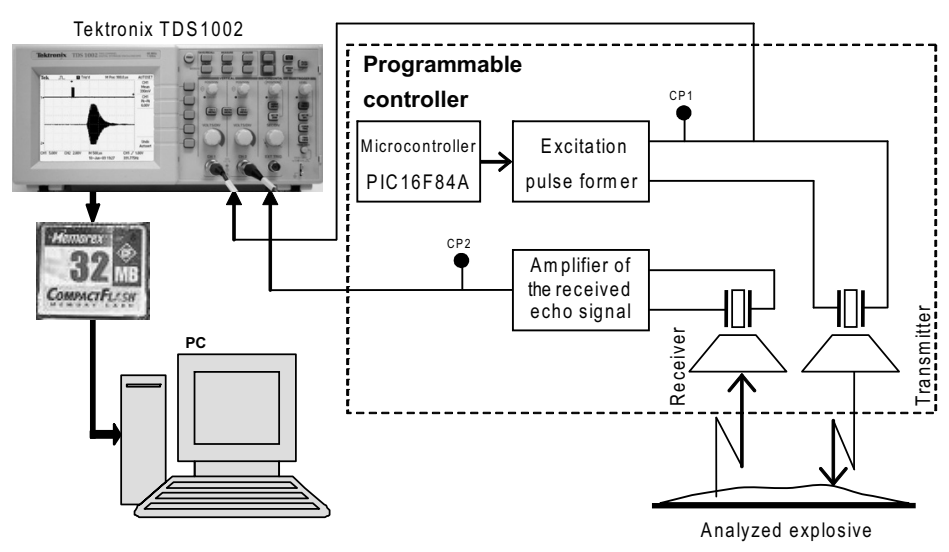

Fig. 1. Block diagram of the system for determining the characteristics of various materials.

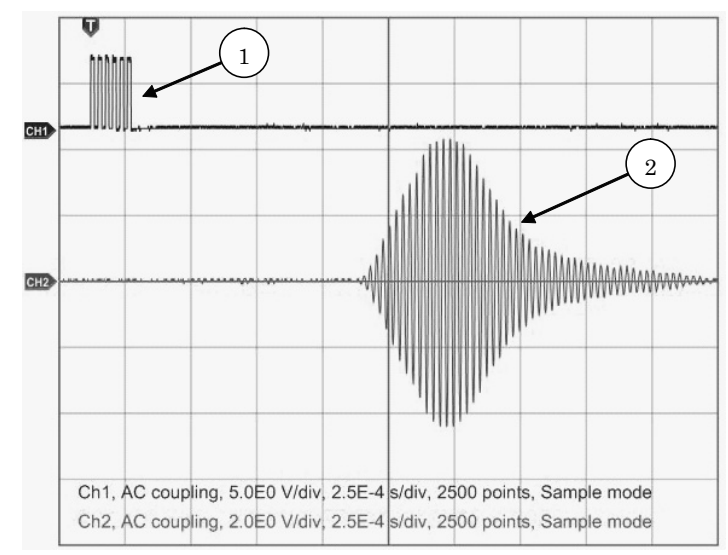

Fig. 2. Oscillogram of the signals: (1) packet of six pulses and (2) reflected echo signal.

whereby the output description $f(t)$ is transformed into a new space with features, namely, the coefficient $S(k)$ of the decomposition of $f(t)$ on a suitable basis:

$$
S(k)=\Phi \cdot f(t)
$$

where $\Phi$ is a transformant.

The last 20 years have witnessed the beginning and active development of the theoretical basis of wavelets and the active work carried out on their application in various fields of technology, including the recognition of random signals. ${ }^{(5,10,12)}$

Wavelets are functions of various shapes that are localized along the independent variable $(t)$ axis, capable of shifting along it and scaling (shrinkage/extension), and having the form of short-wave packets of zero mean value of the wavelet function $\psi(t)$, i.e., ${ }^{(1,2,7,10,12)}$ 


$$
\int_{-\infty}^{\infty} \psi(t) \mathrm{d} t=0
$$

The bases of the wavelet transform are two continuous and integrable functions along the independent variable axis:

- $\psi(t)$ : wavelet function displaying the signal detail and forming the detailing coefficients;

- $\varphi(t)$ : scaling function determining the signal approximation and forming the approximating coefficients. $\varphi$-functions are only characteristic of orthogonal wavelets - e.g., Haar, Daubechies, and Coiflets. ${ }^{(1,2,7,10)}$

The basis function $\psi_{0}(t)$ has to satisfy eq. (2) and the execution of the following operations:

- shift along the independent variable axis:

$$
\psi_{0}(t-b)
$$

where $b$ is a real number $(b \in \mathbb{R})$ determining the position of the wavelet packet;

- scaling:

$$
a^{-1 / 2} \psi_{0}\left(\frac{t}{a}\right)
$$

where $a \in \mathbb{R}, a>0$ determines the width of the wavelet packet.

Taking into account the requirements posed by eqs. (3) and (4),

$$
\psi(t)=a^{-1 / 2} \psi_{0}\left(\frac{t-b}{a}\right) .
$$

For discrete values: $a=2^{m}$ and $b=k 2^{m}$, where $k$ and $m$ are integers, the dependence eq. (5) takes the form

$$
\psi_{m, k}(t)=2^{-m / 2} \psi_{0}\left(2^{-m} t-k\right)
$$

The approximating $A_{m, k}$ and detailing $D_{m, k}$ coefficients of the direct discrete wavelet transform at the level $\mathrm{m}$ are calculated according to the following equations: ${ }^{(5,7,10)}$

$$
\begin{aligned}
& A_{m, k}=\int_{-\infty}^{\infty} 2^{-m / 2} \varphi_{0}\left(2^{-m} t-k\right) f(t) \mathrm{d} t, \\
& D_{m, k}=\int_{-\infty}^{\infty} 2^{-m / 2} \psi_{0}\left(2^{-m} t-k\right) f(t) \mathrm{d} t .
\end{aligned}
$$

In the case of discrete values, the output signal at the level $m$ (as shown in Fig. 3) can be presented using eq. $(2)^{(5,7,10)}$ as 


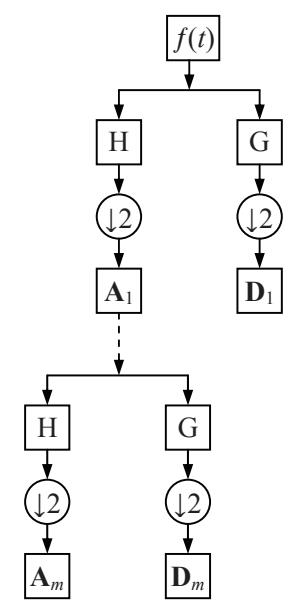

Fig. 3. Diagram of fast wavelet conversion at the level $m$.

$$
f(t)=\sum_{k} A_{m, k} \varphi_{m, k}(t) \sum_{j=1}^{m} \sum_{k} D_{j, k} \psi_{j, k}(t)
$$

For a transformation to be applicable to spectral methods of signal recognition, it must have an orthogonal system of basis functions and there must be fast conversion algorithms. ${ }^{(5,7,10)}$ What is particularly suitable for purposes of automatic classification is the discrete wavelet transform (DWT) with an elaborate fast wavelet conversion, also known as the Mallat pyramidal algorithm - see Fig. 3. $\cdot^{(1,5,7,10)}$

The signal $f(t)$ is passed to a low-frequency $H$ filter with the transfer functions eq. (10) and a high-frequency $G$ filter (Fig. 3) with the transfer functions eq. (11), which are specific for every wavelet: ${ }^{(1,7)}$

$$
\begin{aligned}
& H(\omega)=\sum_{n \in Z} h_{n} e^{-i n \omega}, \\
& G(\omega)=\sum_{n \in Z} g_{n} e^{-i n \omega},
\end{aligned}
$$

corresponding to $\psi(t)$ and $\varphi(t)$.

The filter coefficients $h_{n}$ and $g_{n}$ are calculated depending on the wavelet, and $n$ is an integer. After reducing the number of frequency components in half (binary decimation $(\downarrow 2))$, the approximation coefficients are obtained at the level $m=1, \mathrm{~A}_{1}$ from filter $H$, whereas the detail coefficient $D_{1}$ is obtained from filter $G$. In the case of decomposition at a higher level, the approximation coefficients at the level $m=1\left(A_{1}\right)$ undergo analogous operations according to the results shown in Fig. 3.

In this study, we use a sample of data concerning the values of reflected signals of 690 specimens of Ammonite_ZH-B-E, Ammonite_E, and Trotyl. Table 1 shows the analyzed materials with their class number corresponding to each of them. 
Table 1

Analyzed materials with their corresponding class number.

\begin{tabular}{cc}
\hline Class number, $i$ & Analyzed material \\
\hline 1 & Ammonite_ZH-B-E \\
2 & Ammonite E \\
3 & Trotyl \\
\hline
\end{tabular}

The volume of the output sample is 690 occurrences, broken down as follows - first class, 220; second class, 230; and third class, 240 occurrences. Each occurrence of the echo signal consists of 2500 values recorded when reading the output data. Figure 4 shows the type of reflected signals for the three types of material. Each of them carries specific information on the corresponding substance. The tests have been conducted using air as a working medium at a distance of $0.6 \mathrm{~m}$ from the material.

The training sample is formed from the output data by the random selection of the numbers of occurrences, as follows - first class, 90; second class, 120; and third class, 130 values. The validation set is composed of occurrences that are not included in the training sample.

By the simulation using Matlab, the approximation and detail coefficients of the occurrences in the training sample have been obtained (340 occurrences) at the levels $m=1$ through $m=10$, while applying DWT with orthogonal Haar (Daubechies 1), Daubechies, Coiflets, and Symlet wavelets. The obtained approximation and detail coefficients have been studied in the capacity of recognition (classification) features. At the same time, feature space is reduced. As can be seen in Figs. 5 and 6, the features are 28 at the level $m=7$, and they are reduced to 18 at the level $m=8$.

\section{Results}

The main advantage of the applied approach lies in the possibility of obtaining the features in a strictly defined mathematical procedure, while avoiding the subjective factor in the heuristic formation of features.

The best results have been obtained when applying Haar (Daubechies 1) and Symlet 5 wavelets and using approximation coefficients at the decomposition levels $m=7$ and 8 in the capacity of classification features. The absolute values of the features have been used.

Figures 5 and 6 show some of the features obtained for the three classes of explosives and their average values for Symlet 5 wavelet at the levels $m=7$ and 8 , respectively, while Fig. 7 shows some of the obtained features and their average values for Haar (Daubechies 1) wavelet at the level $m=7$.

After obtaining the classification features, an additional reduction of the classification features space ${ }^{(4,8)}$ was performed using the successive elimination method. From the initial set, $k$ features are eliminated one by one, whereas classification by the KNN method is performed with the remaining set.

Each step eliminates the feature that causes a higher total classification error E. The chosen classifier operates using the $k$-nearest neighbor (KNN) method, taking into 


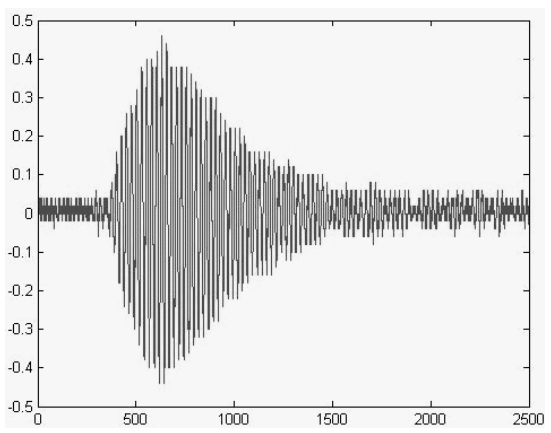

(a)

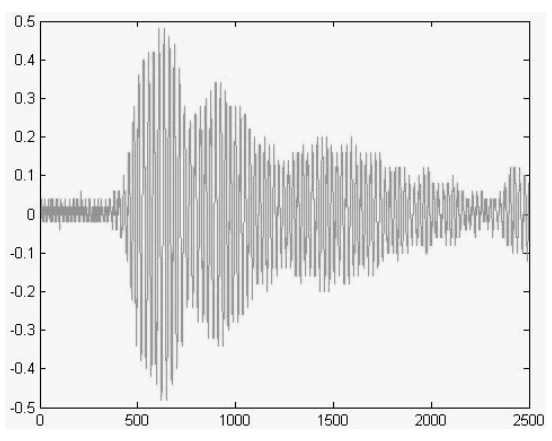

(b)

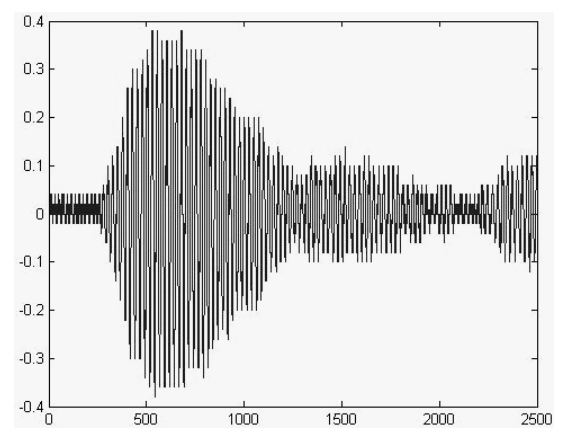

(c)

Fig. 4. Reflected echo signal: (a) Ammonite_ZH-B-E, (b) Ammonite_E, and (c) Trotyl.

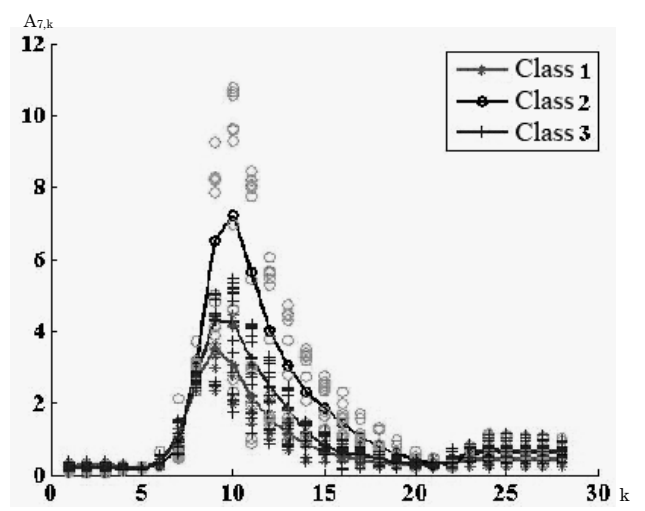

Fig. 5. Values of classification features (approximation coefficients) for wavelet Symlet 5 at decomposition level $m=7$. 


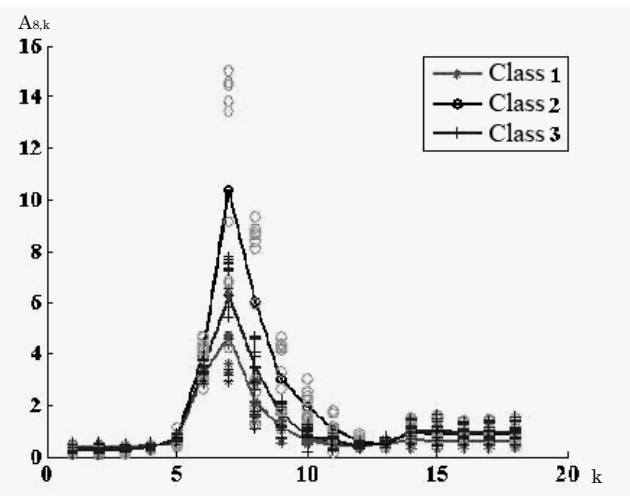

Fig. 6. Values of classification features (approximation coefficients) for wavelet Symlet 5 at decomposition level $m=8$.

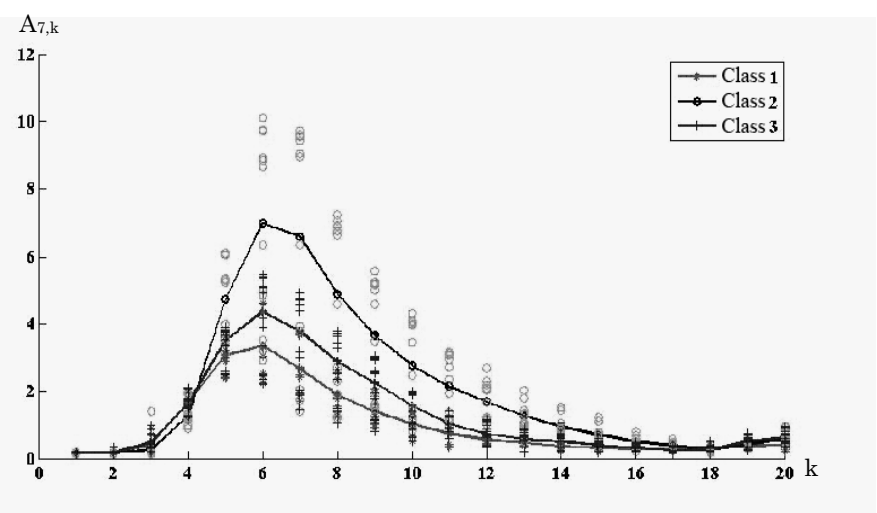

Fig. 7. Values of classification features (approximation coefficients) for Haar wavelet at decomposition level $m=7$.

account the Euclidean distance to the three nearest neighbours. ${ }^{(4,8)}$ The condition for ending the procedure is the achievement of the lowest possible E. By using the feature space formed in this way and the chosen classifier, the validation sample has been classified, with the best results achieved presented in Tables 2-4.

The classification errors are formed as follows:

- The major error $e_{i}$ shows the relative share of the objects of a certain class $i$ related incorrectly by the classifier to the other classes $k=1 \ldots N$ :

$$
e_{i}=\frac{\sum_{k=1}^{N} m_{i k}-m_{i i}}{\sum_{k=1}^{N} m_{i k}} \times 100(\%)
$$


Table 2

Results of classification of validation set (350 specimens) with Symlet 5 wavelet, at level $m=7$ with features $A_{7, k}, k=7,10,11,13,14,16,24,25,26$, and 27.

\begin{tabular}{|c|c|c|c|c|c|c|c|}
\hline \multirow{3}{*}{$\begin{array}{l}\text { Analyzed } \\
\text { material }\end{array}$} & & \multicolumn{4}{|c|}{ Classified by the classifier, number } & \multicolumn{2}{|c|}{ Error } \\
\hline & & $\begin{array}{c}\text { Ammonite } \\
\text { ZH_B-E }\end{array}$ & Ammonite_E & Trotyl & Total & Actual & Major \\
\hline & $m_{i k}$ & $m_{i 1}$ & $m_{i 2}$ & $m_{i 3}$ & & $g_{i}(\%)$ & $e_{i}(\%)$ \\
\hline $\begin{array}{l}\text { Ammonite } \\
\text { ZH_B-E }\end{array}$ & $m_{1 k}$ & 100 & 10 & 20 & 130 & 16.67 & 23.08 \\
\hline $\begin{array}{c}\text { Ammonite } \\
\mathrm{E}\end{array}$ & $m_{2 k}$ & 20 & 90 & 0 & 110 & 10 & 18.18 \\
\hline Trotyl & $m_{3 k}$ & 0 & 0 & 110 & 110 & 15.38 & 0 \\
\hline Total & & 120 & 100 & 130 & 350 & \multicolumn{2}{|c|}{$\begin{array}{l}\text { Total error } \\
E=14.28\end{array}$} \\
\hline
\end{tabular}

Table 3

Results of classification of validation set (350 specimens) with Symlet 5 wavelet, at level $m=8$ with features $\mathrm{A}_{7, k}, k=9,10,11,12,13,14,15,16$, and 17 .

\begin{tabular}{|c|c|c|c|c|c|c|c|}
\hline \multirow{3}{*}{$\begin{array}{l}\text { Analyzed } \\
\text { material }\end{array}$} & & \multicolumn{4}{|c|}{ Classified by the classifier, number } & \multicolumn{2}{|c|}{ Error } \\
\hline & & $\begin{array}{l}\text { Ammonite } \\
\text { ZH_B-E }\end{array}$ & Ammonite_E & Trotyl & Total & Actual & Major \\
\hline & $m_{i k}$ & $m_{i 1}$ & $m_{i 2}$ & $m_{i 3}$ & & $g_{i}(\%)$ & $e_{i}(\%)$ \\
\hline $\begin{array}{l}\text { Ammonite } \\
\text { ZH_B-E }\end{array}$ & $m_{1 k}$ & 100 & 0 & 30 & 130 & 0 & 23.08 \\
\hline $\begin{array}{c}\text { Ammonite } \\
\text { E }\end{array}$ & $m_{2 k}$ & 0 & 90 & 20 & 110 & 0 & 18.18 \\
\hline Trotyl & $m_{3 k}$ & 0 & 0 & 110 & 110 & 31.25 & 0 \\
\hline Total & & 100 & 90 & 160 & 350 & \multicolumn{2}{|c|}{$\begin{array}{l}\text { Total error } \\
E=14.28\end{array}$} \\
\hline
\end{tabular}

\section{Table 4}

Results of classification of validation set (350 specimens) with Haar wavelet (Daubechies 1), at level $m=7$ with features $\mathrm{A}_{7, k}, k=5,10,13,16,17,18$, and 20 .

\begin{tabular}{|c|c|c|c|c|c|c|c|}
\hline \multirow{3}{*}{$\begin{array}{c}\text { Analyzed } \\
\text { material }\end{array}$} & & \multicolumn{4}{|c|}{ Classified by the classifier, number } & \multicolumn{2}{|c|}{ Error } \\
\hline & & $\begin{array}{l}\text { Ammonite } \\
\text { ZH_B-E }\end{array}$ & Ammonite_E & Trotyl & Total & Actual & Major \\
\hline & $m_{i k}$ & $m_{i 1}$ & $m_{i 2}$ & $m_{i 3}$ & & $g_{i}(\%)$ & $e_{i}(\%)$ \\
\hline $\begin{array}{l}\text { Ammonite } \\
\text { ZH_B-E }\end{array}$ & $m_{1 k}$ & 130 & 0 & 0 & 130 & 0 & 0 \\
\hline $\begin{array}{c}\text { Ammonite } \\
\text { E }\end{array}$ & $m_{2 k}$ & 0 & 103 & 7 & 110 & 0 & 6.36 \\
\hline Trotyl & $m_{3 k}$ & 0 & 0 & 110 & 110 & 5.98 & 0 \\
\hline Total & & 130 & 103 & 117 & 350 & \multicolumn{2}{|c|}{$\begin{array}{c}\text { Total error } \\
E=2 \%\end{array}$} \\
\hline
\end{tabular}


- The actual error $g_{i}$ shows the relative share of the objects of other classes $k=1 \ldots N$ related incorrectly by the classifier to a certain class $i$ :

$$
g_{i}=\frac{\sum_{i=1}^{N} m_{i k}-m_{i i}}{\sum_{i=1}^{N} m_{i k}} \times 100(\%) .
$$

- The total error $E$ shows the incorrectly classified objects relative to all objects in the sample:

$$
e_{0}=\frac{\sum_{i=1}^{N}\left(\sum_{k=1}^{N} m_{i k}-m_{i i}\right)}{\sum_{i=1}^{N} \sum_{k=1}^{N} m_{i k}} \times 100(\%),
$$

where $m_{i k}$ is the number of class $i$ specimens related to class $k$ by the classifier, $m_{i i}$ is the number of objects that have been recognized correctly, and $N$ is the number of classes $(N=3)$.

\section{Discussion}

The reflected signal contains information on the tested material. The signal characteristics depend on the substance structure. The differences between the individual signals of the given materials allow them to be studied, classified, and identified.

As a result of studies conducted using the classifiers thus synthesized, the possible classification of three explosives by a noncontact ultrasound method has been proved experimentally; the total errors $\mathrm{E}$ obtained are less than $15 \%$, i.e., a precision above $85 \%$ has been achieved. The highest precision is achieved with a KNN classifier, operating with 7 features formed with the Haar wavelet, i.e., 98\% (Table 4). Ammonite ZH-B-E $(n=130)$ and Trotyl $(n=110)$ specimens have been classified correctly and only 7 of the 110 analyzed Ammonite_E specimens have been recognized as Trotyl.

The conducted classification yields a very high precision (i.e., 100\%) in Trotyl recognition (class 3 ) for both wavelets.

Using the same approach in our other research studies, we have analyzed four types of metal (aluminium, chromium-nickel steel, copper, and brass) and the fat content of yoghurt, and the obtained results yield a recognition precision above $98 \%$.

According to the review in the paper ${ }^{(12)}$ (52 titles on wavelet application), no similar approach to the use of wavelet functions for material recognition has been published.

\section{Conclusions}

The possibility of adapting discrete wavelet transform (DWT) to spectral methods for material recognition in explosive classification problems has been proved experimentally. The use of DWT with a suitable wavelet at a suitable decomposition level has good prospects in explosive classification. 
In view of the methods of noncontact and fast acquisition of information, and the fast wavelet transform and KNN classifier that have been used, the recognition of the test materials can be performed in real time (on-line).

\section{References}

1 F.-T. Chau, Y. Liang, J. Gao and X.-G. Shao: Chemometrics from Basics to Wavelet Transform (John Wiley \& Sons, Inc., Hoboken, 2004).

2 I. Daubechies: Ten Lectures on Wavelets (CBMS-NSF Conference Series in Applied Mathematics, SIAM Ed., 1992).

3 Air Transmission Ultrasonic Sensor (2002, Nippon Ceramic Co., Ltd., http://www.nicera. co.jp/pro/ut/pdf/pdfut002.pdf, 2009).

4 J. P. Marques de Sa: Pattern Recognition. Concepts, Methods, and Applications (Springer-Verlag Berlin Heidelberg, 2001).

5 R. J. E. Merry: Wavelet Theory and Applications (A literature study, Eindhoven University of Technology, Eindhoven, 2005, http://alexandria.tue.nl/repository/books/612762.pdf).

6 L. Thiesan, D. Hannum, D. W. Murray and J. E. Parmeter: Survey of Commercially Available Explosives Detection Technologies and Equipment (2004, www.ncjrs.gov/pdffiles1/nij/ grants/208861.pdf).

7 Wavelet Toolbox User's Guide (The MathWorks Inc., 2002).

8 A. Webb: Statistical Pattern Recognition, 2nd ed. (John Wiley \& Sons, Hoboken, 2002).

9 American Innovations, Inc. (2009, www.explosivesdetection.com).

10 S. Mallat: IEEE Trans. Pattern Anal. Mach. Intell. 11 (1989) 674.

11 H. Baltes, W. Göpel and J. Hesse: Sensors Update. Sensor Technology-Applications-Markets (Wiley-VCH Verlag GmbH, Weinheim, 1998) Vol. 3.

12 C. Gargour, M. Gabrea, V. Ramachandran and J. Lina: A short introduction to wavelets and their application, IEEE Circuits and Systems Magazine, Second Quarter 2009, pp. 57-68. 